Bull. Korean Math. Soc. 51 (2014), No. 4, pp. 1187-1193

http://dx.doi.org/10.4134/BKMS.2014.51.4.1187

\title{
WEIGHTED COMPOSITION OPERATORS ON THE MINIMAL MÖBIUS INVARIANT SPACE
}

\author{
SHÛICHI OHNO
}

ABstract. We will characterize the boundedness and compactness of weighted composition operators on the minimal Möbius invariant space.

\section{Introduction}

Here and henceforth, $\mathbb{D}$ will denote the open unit disk $\mathbb{D}:=\{z \in \mathbb{C}:|z|<1\}$. The set of all conformal automorphisms of $\mathbb{D}$ forms a group, called a Möbius group and denoted by $\operatorname{Aut}(\mathbb{D})$. For any $\lambda \in \mathbb{D}$, let

$$
\alpha_{\lambda}(z)=\frac{\lambda-z}{1-\bar{\lambda} z}
$$

be the Möbius transformation of $\mathbb{D}$. Let $X$ be a linear space of analytic functions on $\mathbb{D}$. Then $X$ is said to be Möbius invariant if $f \circ \alpha \in X$ for all $f \in X$ and all $\alpha \in \operatorname{Aut}(\mathbb{D})$. A typical example of Möbius invariant spaces is the Besov space. For $1<p<\infty$, let $B_{p}$ be the space of analytic functions $f$ on $\mathbb{D}$ such that

$$
\int_{\mathbb{D}}\left|f^{\prime}(z)\right|^{p}(1-|z|)^{p-2} d A(z)<\infty
$$

where $d A$ is the normalized Lebesgue area measure on $\mathbb{D}$. Then $B_{p}$ is the Banach space with the norm

$$
\|f\|_{B_{p}}=|f(0)|+\left(\int_{\mathbb{D}}\left|f^{\prime}(z)\right|^{p}(1-|z|)^{p-2} d A(z)\right)^{1 / p} .
$$

If $p=2, B_{2}$ is the classical Dirichlet space that is minimal as Möbius invariant Hilbert space of analytic functions on $\mathbb{D}$. The analytic Besov space $B_{1}$ is the

Received October 14, 2013; Revised January 7, 2014.

2010 Mathematics Subject Classification. Primary 47B38; Secondary 30H10.

Key words and phrases. weighted composition operator, Besov spaces, the minimal Möbius invariant space.

The author is partially supported by Grant-in-Aid for Scientific Research, Japan Society for the Promotion of Science (No.24540190). 
space of all analytic functions $f$ for which

$$
f(z)=\sum_{n=1}^{\infty} a_{n} \alpha_{\lambda_{n}}(z),
$$

for some sequence $\left\{a_{n}\right\} \in \ell^{1}$ and $\left\{\lambda_{n}\right\}$ in $\mathbb{D}$. Then the norm $\|f\|_{B_{1}}$ is defined by

$$
\|f\|_{B_{1}}=\inf \left\{\sum_{n=1}^{\infty}\left|a_{n}\right|: f(z)=\sum_{n=1}^{\infty} a_{n} \alpha_{\lambda_{n}}(z)\right\} .
$$

It is known that $B_{1}$ is minimal, as it is contained in any Möbius invariant space and that the norm $\|f\|_{B_{1}}$ is equivalent to

$$
|f(0)|+\left|f^{\prime}(0)\right|+\int_{\mathbb{D}}\left|f^{\prime \prime}(z)\right| d A(z) .
$$

For the study of Besov spaces one can refer to $[1,2,12,13]$ and references therein.

Let $u$ be a fixed analytic function on $\mathbb{D}$ and $\varphi$ an analytic self-map of $\mathbb{D}$. Then the weighted composition operator $u C_{\varphi}$ is defined by

$$
\left(u C_{\varphi}\right) f=u \cdot f \circ \varphi
$$

for analytic functions $f$ on $\mathbb{D}$. In these five decades, there has been much work on weighted composition operators on various spaces of analytic functions on $\mathbb{D}$. See $[6,8]$ for an overview of these results.

Composition operators between the Besov spaces have been investigated since Tjani [9] studied. Those operators on the minimal Möbius invariant subspace $B_{1}$ also have been studied. For example, see $[3,10]$. In particular, Wulan and Xiong [10] proved that the compactness criterion of composition operators on $B_{p}(1<p<\infty)$, which is Tjani's result [9], still holds for $B_{1}$. Furthermore, composition operators from the Besov spaces to any analytic function space have been characterized in [11]. Recently it is given the characterization of the weighted composition operators mapping the Besov spaces to the Bloch space in $[4,5]$. However properties of each weighted composition operator acting from $B_{1}$ to $B_{1}$ are left behind. We here carry on studying this problem. That is, we will characterize the boundedness and compactness of weighted composition operators mapping the minimal Möbius invariant space $B_{1}$ to $B_{1}$.

\section{Boundedness and compactness on $B_{1}$}

In order to characterize boundedness and compactness on $B_{1}$, we introduce the new generalized integral type operators.

Let $u$ be a fixed analytic function on $\mathbb{D}$ and $\varphi$ an analytic self-map of $\mathbb{D}$. Then we define

$$
C_{\varphi}^{u} f(z)=\int_{0}^{z}(f \circ \varphi)^{\prime}(\zeta) u(\zeta) d \zeta
$$


and

$$
D_{\varphi}^{u} f(z)=\int_{0}^{z}(f \circ \varphi)(\zeta) u^{\prime}(\zeta) d \zeta
$$

for analytic functions $f$ on $\mathbb{D}$.

If $u \equiv 1$, then

$$
C_{\varphi}^{u} f(z)=(f \circ \varphi)(z)-f(\varphi(0))=C_{\varphi} f(z)-f(\varphi(0)) \quad \text { and } \quad D_{\varphi}^{n} f \equiv 0 .
$$

If $\varphi(z) \equiv z$, then

$$
C_{\varphi}^{u} f(z)=\int_{0}^{z} f^{\prime}(\zeta) u(\zeta) d \zeta
$$

and

$$
D_{\varphi}^{u} f(z)=\int_{0}^{z} f(\zeta) u^{\prime}(\zeta) d \zeta
$$

At first we have the result on the boundedness of $u C_{\varphi}$ on $B_{1}$.

Proposition 2.1. Let $u$ be a fixed analytic function on $\mathbb{D}$ and $\varphi$ an analytic self-map of $\mathbb{D}$. Then the following are equivalent.

(i) $u C_{\varphi}$ is bounded on $B_{1}$.

(ii) $\sup _{\lambda \in \mathbb{D}}\left\|u C_{\varphi} \alpha_{\lambda}\right\|_{B_{1}}<\infty$.

(iii) $\sup _{\lambda \in \mathbb{D}}\left\|\left(C_{\varphi}^{u}+D_{\varphi}^{u}\right) \alpha_{\lambda}\right\|_{B_{1}}<\infty$.

Proof. The equivalence of (i) and (ii) is trivial. As

$$
\left(u C_{\varphi} \alpha_{\lambda}\right)^{\prime \prime}=\left(\left(C_{\varphi}^{u}+D_{\varphi}^{u}\right) \alpha_{\lambda}\right)^{\prime \prime},
$$

we obtain the equivalence of (ii) and (iii).

In the proof of characterization of compact (weighted) composition operators we usually need the so-called "weak convergence theorem", which we can show by the similar way as in the proof of Proposition 3.11 in [6].

Proposition 2.2. Let $u$ be a fixed analytic function on $\mathbb{D}$ and $\varphi$ an analytic self-map of $\mathbb{D}$. Suppose that $u C_{\varphi}$ is bounded on $B_{1}$. Then $u C_{\varphi}$ is compact on $B_{1}$ if and only if $\left\|u C_{\varphi} f_{n}\right\|_{B_{1}} \rightarrow 0$ as $n \rightarrow \infty$ for every sequence $\left\{f_{n}\right\}_{n}$ in $B_{1}$ with $\left\|f_{n}\right\|_{B_{1}} \leq 1$ satisfying $f_{n} \rightarrow 0$ uniformly on compact subsets of $\mathbb{D}$.

Thus we could characterize the compactness.

Theorem 2.3. Let $u$ be a fixed analytic function on $\mathbb{D}$ and $\varphi$ an analytic selfmap of $\mathbb{D}$ with $\|\varphi\|_{\infty}=1$. Suppose that $u C_{\varphi}$ is bounded on $B_{1}$. Then the following are equivalent.

(i) $u C_{\varphi}$ is compact on $B_{1}$.

(ii) $\lim _{|\lambda| \rightarrow 1}\left\|u C_{\varphi}\left(\alpha_{\lambda}-\lambda\right)\right\|_{B_{1}}=0$.

(iii) $\lim _{|\lambda| \rightarrow 1}\left\|\left(C_{\varphi}^{u}+D_{\varphi}^{u}\right)\left(\alpha_{\lambda}-\lambda\right)\right\|_{B_{1}}=0$.

(iv) $\lim _{r \rightarrow 1} \sup _{\lambda \in \mathbb{D}} \int_{\{|\varphi(z)|>r\}}\left|\left(u C_{\varphi}\left(\alpha_{\lambda}-\lambda\right)\right)^{\prime \prime}(z)\right| d A(z)=0$. 
Proof. At first, by the boundedness we can note that $u^{\prime \prime}, 2 u^{\prime} \varphi^{\prime}+u \varphi^{\prime \prime}$ and $u\left(\varphi^{\prime}\right)^{2}$ are $L^{1}(\mathbb{D})$-summable since $u C_{\varphi} 1, u C_{\varphi} z$ and $u C_{\varphi} z^{2}$ are in $B_{1}$.

The implication (i) $\Rightarrow$ (ii) is shown because $\alpha_{\lambda}-\lambda$ converges to 0 uniformly on compact subsets of $\mathbb{D}$ as $|\lambda| \rightarrow 1$. The equivalence of (ii) and (iii) is implied since $\left(u C_{\varphi}\left(\alpha_{\lambda}-\lambda\right)\right)^{\prime \prime}=\left(\left(C_{\varphi}^{u}+D_{\varphi}^{u}\right)\left(\alpha_{\lambda}-\lambda\right)\right)^{\prime \prime}$.

Next we will prove the implication (ii) $\Rightarrow$ (iv). By (ii), for any $\varepsilon>0$, there exists a constant $\delta, 0<\delta<1$, such that

$$
\sup _{|\lambda|>\delta} \int_{\mathbb{D}}\left|\left(u C_{\varphi}\left(\alpha_{\lambda}-\lambda\right)\right)^{\prime \prime}(z)\right| d A(z)<\varepsilon .
$$

Moreover for all $r, 0<r<1$,

On the other hand,

$$
\sup _{|\lambda|>\delta} \int_{\{|\varphi(z)|>r\}}\left|\left(u C_{\varphi}\left(\alpha_{\lambda}-\lambda\right)\right)^{\prime \prime}(z)\right| d A(z)<\varepsilon .
$$

$$
\begin{aligned}
& \sup _{|\lambda| \leq \delta} \int_{\{|\varphi(z)|>r\}}\left|\left(u C_{\varphi}\left(\alpha_{\lambda}-\lambda\right)\right)^{\prime \prime}(z)\right| d A(z) \\
& =\sup _{|\lambda| \leq \delta} \int_{\{|\varphi(z)|>r\}} \mid u^{\prime \prime}(z)\left(\alpha_{\lambda}(\varphi(z))-\lambda\right) \\
& +2 u^{\prime}(z) \alpha_{\lambda}^{\prime}(\varphi(z)) \varphi^{\prime}(z)+u(z) \alpha_{\lambda}^{\prime \prime}(\varphi(z))\left(\varphi^{\prime}(z)\right)^{2} \\
& +u(z) \alpha_{\lambda}^{\prime}(\varphi(z)) \varphi^{\prime \prime}(z) \mid d A(z) \\
& \leq C\left(\int_{\{|\varphi(z)|>r\}}\left|u^{\prime \prime}(z)\right| d A(z)\right. \\
& +\int_{\{|\varphi(z)|>r\}}\left|2 u^{\prime}(z) \varphi^{\prime}(z)+u(z) \varphi^{\prime \prime}(z)\right| d A(z) \\
& \left.+\int_{\{|\varphi(z)|>r\}}\left|u(z)\left(\varphi^{\prime}(z)\right)^{2}\right| d A(z)\right),
\end{aligned}
$$

where $C=\max \left\{2, \sup \left\{\left|\alpha_{\lambda}^{\prime}(\varphi(z))\right|:|\lambda| \leq \delta, z \in \mathbb{D}\right\}, \sup \left\{\left|\alpha_{\lambda}^{\prime \prime}(\varphi(z))\right|:|\lambda| \leq\right.\right.$ $\delta, z \in \mathbb{D}\}$. Considering that $u^{\prime \prime}, 2 u^{\prime} \varphi^{\prime}+u \varphi^{\prime \prime}$ and $u\left(\varphi^{\prime}\right)^{2}$ are $L^{1}(\mathbb{D})$-summable,

$$
\lim _{r \rightarrow 1} \sup _{|\lambda| \leq \delta} \int_{\{|\varphi(z)|>r\}}\left|\left(u C_{\varphi}\left(\alpha_{\lambda}-\lambda\right)\right)^{\prime \prime}(z)\right| d A(z)=0 .
$$

Consequently,

$$
\begin{aligned}
& \quad \lim _{r \rightarrow 1} \sup _{\lambda \in \mathbb{D}} \int_{\{|\varphi(z)|>r\}}\left|\left(u C_{\varphi} \alpha_{\lambda}\right)^{\prime \prime}(z)\right| d A(z) \\
& \leq \lim _{r \rightarrow 1} \sup _{|\lambda| \leq \delta} \int_{\{|\varphi(z)|>r\}}\left|\left(u C_{\varphi} \alpha_{\lambda}\right)^{\prime \prime}(z)\right| d A(z) \\
& \quad+\lim _{r \rightarrow 1} \sup _{|\lambda|>\delta} \int_{\{|\varphi(z)|>r\}}\left|\left(u C_{\varphi} \alpha_{\lambda}\right)^{\prime \prime}(z)\right| d A(z) \\
& <\varepsilon .
\end{aligned}
$$


As $\varepsilon$ is arbitrary,

$$
\lim _{r \rightarrow 1} \sup _{\lambda \in \mathbb{D}} \int_{\{|\varphi(z)|>r\}}\left|\left(u C_{\varphi} \alpha_{\lambda}\right)^{\prime \prime}(z)\right| d A(z)=0 .
$$

So we obtain condition (iv).

Finally we show the implication (iv) $\Rightarrow(i)$. By condition (iv) and the $L^{1}(\mathbb{D})$ summability of $u^{\prime \prime}$, for any $\varepsilon>0$, there is a constant $r, 0<r<1$, such that

and

$$
\int_{\{|\varphi(z)|>r\}}\left|\left(u C_{\varphi}\left(\alpha_{\lambda}-\lambda\right)\right)^{\prime \prime}(z)\right| d A(z)<\varepsilon
$$

$$
\int_{\{|\varphi(z)|>r\}}\left|u^{\prime \prime}(z)\right| d A(z)<\varepsilon .
$$

Let $\left\{f_{n}\right\}_{n}$ be a sequence of functions in $B_{1}$ with $\left\|f_{n}\right\|_{B_{1}} \leq 1$ satisfying $f_{n} \rightarrow 0$ uniformly on compact subsets of $\mathbb{D}$. Then we have

$$
f_{n}(z)=\sum_{k=1}^{\infty} a_{n, k} \alpha_{\lambda_{n, k}}(z), \quad \lambda_{n, k} \in \mathbb{D},
$$

with

$$
\left\|f_{n}\right\|_{B_{1}} \leq \sum_{k=1}^{\infty}\left|a_{n, k}\right| \leq 2 .
$$

Trivially $\left|\left(u C_{\varphi} f_{n}\right)(0)\right|+\left|\left(u C_{\varphi} f_{n}\right)^{\prime}(0)\right| \rightarrow 0$ as $n \rightarrow \infty$.

Then

$$
\begin{aligned}
& \left\|u C_{\varphi} f_{n}\right\|_{B_{1}} \\
= & \int_{\mathbb{D}}\left|\left(u C_{\varphi} f_{n}\right)^{\prime \prime}(z)\right| d A(z) \\
= & \int_{\{|\varphi(z)| \leq r\}}\left|\left(u C_{\varphi} f_{n}\right)^{\prime \prime}(z)\right| d A(z)+\int_{\{|\varphi(z)|>r\}}\left|\left(u C_{\varphi} f_{n}\right)^{\prime \prime}(z)\right| d A(z) .
\end{aligned}
$$

Here

$$
\begin{aligned}
& \int_{\{|\varphi(z)|>r\}}\left|\left(u C_{\varphi} f_{n}\right)^{\prime \prime}(z)\right| d A(z) \\
& \leq \int_{\{|\varphi(z)|>r\}}\left|\left(u C_{\varphi}\left(\sum_{k=1}^{\infty} a_{n, k}\left(\alpha_{\lambda_{n, k}}-\lambda_{n, k}\right)\right)\right)^{\prime \prime}(z)\right| d A(z) \\
&+\int_{\{|\varphi(z)|>r\}}\left|\left(u C_{\varphi}\left(\sum_{k=1}^{\infty} a_{n, k} \lambda_{n, k}\right)\right)^{\prime \prime}(z)\right| d A(z) \\
& \leq \sum_{k=1}^{\infty}\left|a_{n, k}\right| \int_{\{|\varphi(z)|>r\}}\left|\left(u C_{\varphi}\left(\alpha_{\lambda_{n, k}}-\lambda_{n, k}\right)\right)^{\prime \prime}(z)\right| d A(z) \\
&+\sum_{k=1}^{\infty}\left|a_{n, k}\right| \int_{\{|\varphi(z)|>r\}}\left|u^{\prime \prime}(z)\right| d A(z)
\end{aligned}
$$




$$
<4 \varepsilon \text {. }
$$

So

$$
\begin{aligned}
\lim _{n \rightarrow \infty}\left\|u C_{\varphi} f_{n}\right\|_{B_{1}} & \leq \lim _{n \rightarrow \infty} \int_{\{|\varphi(z)| \leq r\}}\left|\left(u C_{\varphi} f_{n}\right)^{\prime \prime}(z)\right| d A(z)+4 \varepsilon \\
& =4 \varepsilon .
\end{aligned}
$$

As $\varepsilon$ is arbitrary,

$$
\lim _{n \rightarrow \infty}\left\|u C_{\varphi} f_{n}\right\|_{B_{1}}=0 .
$$

By Proposition 2.2, $u C_{\varphi}$ is compact on $B_{1}$.

Lastly we add some comment in the unweighted case. Since functions in $B_{1}$ extend continuously to the boundary of $\mathbb{D}$, a result of [7] characterizes the compactness of $C_{\varphi}$ on $B_{1}$, so that $C_{\varphi}$ is compact on $B_{1}$ if and only if $\varphi \in B_{1}$ and $\|\varphi\|_{\infty}<1$.

By noticing that $\alpha_{\lambda}^{\prime}$ and $\alpha_{\lambda}^{\prime \prime}$ converge uniformly to 0 on compact subsets of $\mathbb{D}$, we obtain the following $([10])$.

Corollary 2.4. For an analytic self-map $\varphi$ of $\mathbb{D}, C_{\varphi}$ is compact on $B_{1}$ if and only if $\varphi \in B_{1}$ and

$$
\lim _{|\lambda| \rightarrow 1} \int_{\mathbb{D}}\left|\left(C_{\varphi}\left(\alpha_{\lambda}-\lambda\right)\right)^{\prime \prime}(z)\right| d A(z)=\lim _{|\lambda| \rightarrow 1} \int_{\mathbb{D}}\left|\left(\alpha_{\lambda} \circ \varphi\right)^{\prime \prime}(z)\right| d A(z)=0 .
$$

Acknowledgement. We would like to thank the referee for careful reading and honest suggestions that helped correcting some of the proof.

\section{References}

[1] J. Arazy, S. D. Fisher, and J. Peetre, Möbious invariant function spaces, J. Reine Angew. Math. 363 (1985), 110-145.

[2] G. Bao and H. Wulan, The minimal Möbious invariant spaces, Complex Var. Elliptic Equ. 59 (2014), no. 2, 190-203.

[3] O. Blasco, Composition operators on the minimal space invariant under Möbious transformations, Complex and harmonic analysis, 157-166, DEStech Publ. Inc., Lancaster, PA, 2007.

[4] F. Colonna and S. Li, Weighted composition operators from the minimal Möbious invariant space into the Bloch space, Mediterr. J. Math. 10 (2013), no. 1, 395-409.

[5] Weighted composition operators from the Besov spaces into the Bloch spaces, Bull. Malays. Math. Sci. Soc. (2) 36 (2013), no. 4, 1027-1039.

[6] C. C. Cowen and B. D. MacCluer, Composition Operators on Spaces of Analytic Functions, CRC Press, Boca Raton, 1995.

[7] J. H. Shapiro, Compact composition operators on spaces of boundary-regular holomorphic functions, Proc. Amer. Math. Soc. 100 (1987), no. 1, 49-57.

[8] Composition Operators and Classical Function Theory, Springer-Verlag, New York, 1993.

[9] M. Tjani, Compact composition operators on Besov spaces, Trans. Amer. Math. Soc. 355 (2003), no. 11, 4683-4698.

[10] H. Wulan and C. Xiong, Composition operators on the minimal Möbious invariamt space, Hilbert spaces of analytic functions, 203-209, CRM Proc. Lecture Notes, 51, Amer. Math. Soc., Providence, RI, 2010. 
[11] R. Zhao, Composition operators from Bloch type spaces to Hardy and Besov spaces, J. Math. Anal. Appl. 233 (1999), no. 2, 749-766.

[12] K. Zhu, Operator Theory in Function Spaces, Marcel Dekker, New York, 1990; Second Edition, Amer. Math. Soc., Providence, 2007.

[13] _ Analytic Besov spaces, J. Math. Anal. Appl. 157 (1991), no. 2, 318-336.

Nippon Institute of Technology

Miyashiro, Minami-Saitama 345-8501, JaPan

E-mail address: ohno@nit.ac.jp 